\title{
Vaccine-preventable anal infections by Human Papillomavirus among HIV-uninfected men who have sex with men
}

\section{MG Donà ${ }^{1}$, A Latini ${ }^{1}$, M Frasca $^{1}$, M Benevolo $^{2}$, F Rollo², A Giglio ${ }^{3}$,

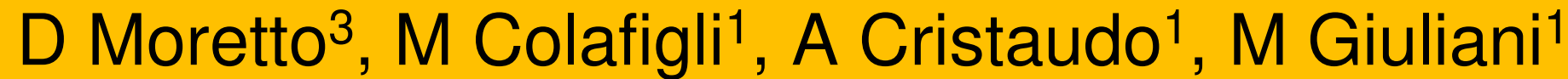

${ }^{1}$ STI/HIV Unit, San Gallicano Dermatologic Institute, IRCCS, Rome, Italy; ${ }^{2}$ Pathology Department, Regina Elena National Cancer Institute, IRCCS, Rome, Italy; ${ }^{3}$ Microbiology and Clinical Pathology Department, S. Gallicano Dermatologic Institute, IRCCS, Rome, Italy

\section{Background}

Men who have sex with men (MSM) are at high risk for anal HPV infection and anal cancer. Two vaccines are available for the prevention of ano-genital warts, anal intraepithelial neoplasia and anal cancer in men: a quadrivalent (4VHPV, HPV 6, 11, 16, 18) and a nonavalent (9vHPV, HPV 6, 11, 16, 18, 31, 33, 45, 52, and 58) HPV vaccine. We estimated the prevalence of the vaccine-preventable HPV genotypes targeted by these vaccines among sexually active HIV-uninfected MSM attending the largest STI centre in Rome (Italy).

\section{Methods}

-Population: HIV-uninfected MSM

-Sample collection: anal samples were collected in PreservCyt® (Hologic) using a Dacron swab

-HPV assay: Linear Array HPV Genotyping Test (Roche Diagnostics)

\begin{tabular}{llcc|}
\multicolumn{4}{l}{ Results } \\
\hline Table 1. Study population (N=443 HIV-uninfected & MSM) \\
\hline & \multicolumn{2}{c}{ Median } & IQR \\
\hline Age, years & 32 & 27 to 39 \\
Age at first sexual intercourse (years) & 19 & 17 to 24 \\
N. of lifetime sexual partners & 50 & 20 to 150 \\
$\begin{array}{l}\text { N. of recent sexual partners } \\
\text { (previous } 6 \text { months) }\end{array}$ & 5 & 2 to 10 \\
\hline
\end{tabular}

Receptive anal intercourse (RAI): 74.0\%

Condomless RAI with occasional partners: $22.4 \%$

Figure 1. Anal HPV prevalence

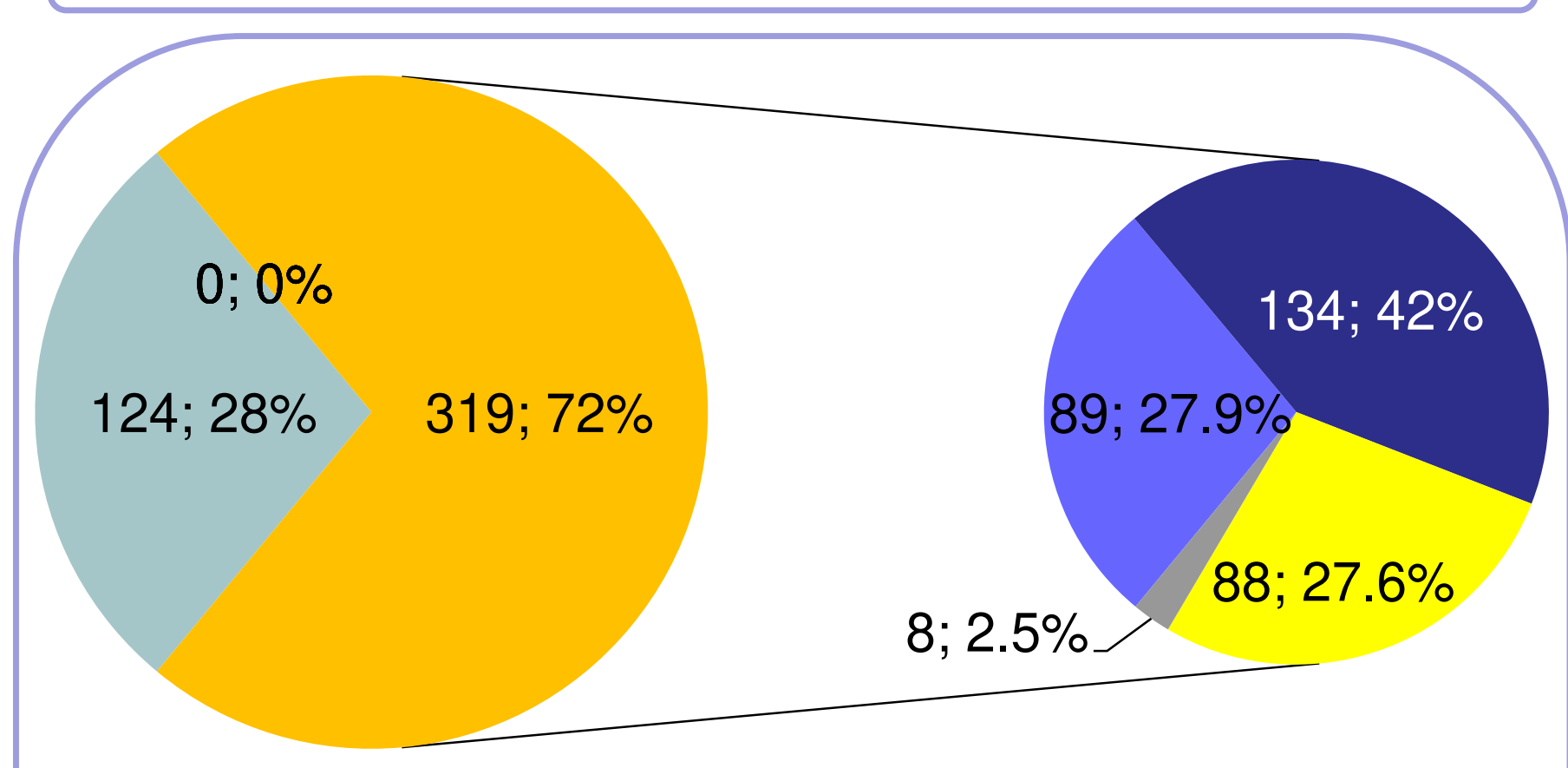

\footnotetext{
HPV-negative

HPV-positive

- High-risk HPVs only

- High-risk + Low-risk HPVs

Low-risk HPVs only

- Intermediate/undetermined risk HPVs only
}

Table 2. HPV infection with the 4vHPV and 9vHPV vaccine types

\begin{tabular}{|c|c|c|c|c|}
\hline & & & $\mathbf{N}$ & $\%$ \\
\hline at least one 4vHPV type & & & 151 & 34.1 \\
\hline at least one 9vHPV type & & & 204 & 46.0 \\
\hline at least one additional 9vHP & pe $(31$ & $5,52,58)$ & 94 & 21.2 \\
\hline \multirow[t]{2}{*}{ No. of HPV vaccine types } & \multicolumn{2}{|c|}{ 4vHPV } & \multicolumn{2}{|c|}{ 9vHPV } \\
\hline & $\mathbf{N}$ & $\%$ & $\mathbf{N}$ & $\%$ \\
\hline 1 & 117 & 26.4 & 135 & 30.5 \\
\hline 2 & 33 & 7.4 & 54 & 12.2 \\
\hline 3 & 1 & 0.2 & 12 & 2.7 \\
\hline 4 & 0 & 0.0 & 2 & 0.5 \\
\hline 5 & - & - & 1 & 0.2 \\
\hline$>5$ & - & - & 0 & 0.0 \\
\hline
\end{tabular}

Figure 2. Prevalence of the $4 \mathrm{vHPV}$ and $9 \mathrm{vHPV}$ vaccine types according to the age-class

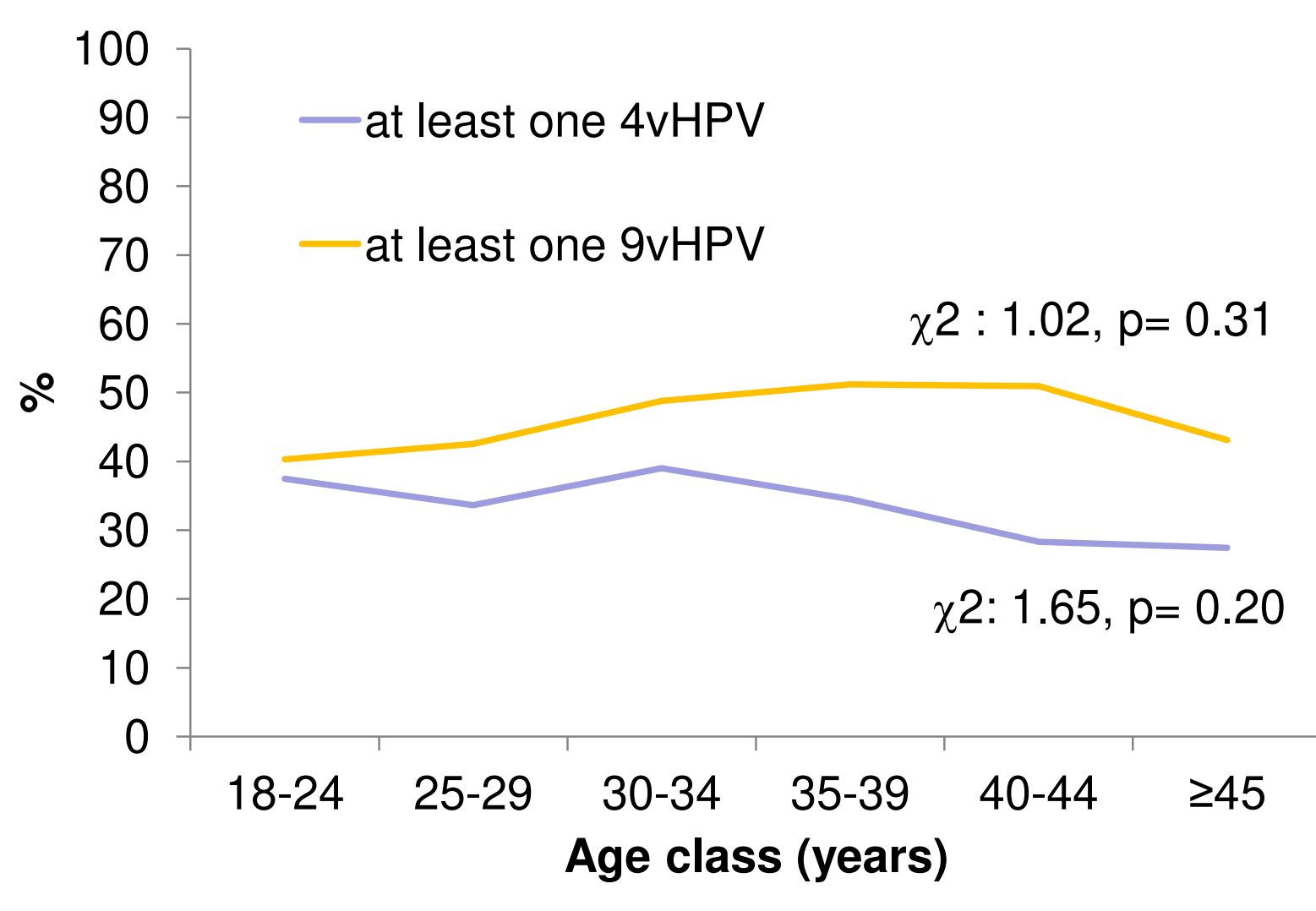

\section{Conclusions}

The prevalence of anal HPV among HIV-uninfected MSM is dramatically high. Nonetheless, around half of the patients were negative for the HPVs included in the HPV vaccines. The prevalence changed significantly by age neither for the 4vHPV nor the 9vHPV vaccine. Although this does not provide information regarding previous infections, a substantial proportion of MSM could benefit from the vaccination with the prophylactic HPV vaccines. The preventive potential of these vaccines in older MSM remains to be determined. 